\title{
Noise characterization of femtosecond color-center lasers
}

\author{
U. Keller, C. E. Soccolich, G. Sucha, M. N. Islam, and M. Wegener
}

AT\&T Bell Laboratories, Crawfords Corner Road, Holmdel, New Jersey 07733

Received March 13, 1990; accepted June 27, 1990

\begin{abstract}
We used power-spectrum techniques to measure both amplitude noise and timing jitter of two femtosecond colorcenter lasers in the near infrared: a passively mode-locked laser and an additive-pulse mode-locked laser. The $\mathrm{cw}$ pumped passively mode-locked color-center laser showed better noise performance than the feedback-controlled, much more complex additive-pulse mode-locked color-center laser. For both laser systems, we observed unexpected drastic increases of amplitude noise under certain operation conditions. In the low-noise operation regime, the amplitude noise of the passively mode-locked color-center laser is entirely dominated by its pump laser, whereas the additive-pulse mode-locked laser exhibits $>10 \mathrm{~dB}$ of excess noise up to $200 \mathrm{kHz}$ and tended to break up in the subhertz regime. The timing jitter for both lasers was approximately 9 psec rms for jitter rates above $130 \mathrm{~Hz}$.
\end{abstract}

Femtosecond pulses in the near infrared, covering the wavelength region that is important for telecommunication, have been generated by a passively modelocked (PM) $\mathrm{NaCl}$ color-center laser ${ }^{1}$ and an additivepulse mode-locked (APM) KCl:Tl color-center laser. ${ }^{2-4}$ The PM color-center laser has the advantage of being a less complex laser system, pumped by a simple $\mathrm{cw}$ $\mathrm{Nd}$ :YAG laser, whereas the APM laser requires synchronous pumping and an active feedback control system. Currently the APM color-center laser typically generates 150-fsec pulses, which are shorter than the 275-fsec PM laser pulses. However, the shorter pulses from the APM laser are gained at the expense of lower stability and higher complexity.

Using power-spectrum techniques, ${ }^{5-7}$ we have measured both the amplitude noise and the timing jitter (fluctuations in the arrival time of the pulses) of both femtosecond color-laser systems. Detailed noisepower-spectrum characterizations give significant information about the sensitivity and time resolution for different measurement applications by simply using a photodetector and a rf spectrum analyzer. Typically, amplitude noise is only measured in the time domain with a detector and a scope, not revealing anything about the noise spectrum of the laser, which often determines the sensitivity (i.e., the minimum detectable signal) of a measurement. For example, laser noise typically decreases with increased frequency, permitting better sensitivity if the measurement can be done at a higher chopping frequency. This was used to improve the sensitivity in electro-optic sampling $^{8}$ so that the minimum detectable signal was set by the shot-noise level. ${ }^{9}$ Therefore, we will give the amplitude noise power as a function of the frequency in the number of decibels above the shot noise at $1 \mathrm{~mA}$ of dc detector photocurrent. It is important to note that this number of decibels above the shot noise scales linearly with the photocurrent, because excess laser noise power measured with a photodetector and a spectrum analyzer scales with the square of the photocurrent, whereas the shot-noise power scales linearly with the photocurrent. While the timing jitter (fluctuations in the arrival time of the laser pulses) is not im portant in pump-probe experiments using the same optical pulse, low timing jitter is essential for experiments involving synchronization of the laser repetition rate with a microwave synthesizer, synchronization of pulses from two different lasers, or synchronization of pulses separated by a longer time duration. The time resolution in the measurement is then given by both the pulse width and the timing jitter integrated over a certain frequency range from $f_{\text {low }}$ to $f_{\text {high }}$, where $f_{\text {low }}$ is determined by the measurement duration and $f_{\text {high }}$ is determined by the detection bandwidth. Typically, the timing jitter sidebands decrease with increased frequency offset. The overall time resolution can be improved with a shorter acquisition time, which, on the other hand, requires good sensitivity.

The higher-frequency amplitude noise was measured with a shot-noise-limited detector, which consists of a $500 \mu \mathrm{m} \times 500 \mu \mathrm{m}$ Ge photodiode with a $1-\mathrm{k} \Omega$ load resistor (which increases the noise voltage due shot noise above the Johnson noise voltage), followed by a dc block and a buffer amplifier (AD LH0033CG) in order to drive the $50-\Omega$ input impedance of the spectrum analyzer. The timing jitter was measured with a fast detector and the rf spectrum analyzer. Note that the timing jitter noise sidebands increase with the square of the laser harmonic and dominate the noise sidebands at sufficiently high harmonics. The timing jitter will be given by the single-sided phase noise (which equals the timing jitter) spectral density of the laser first harmonic $L_{1}(f)$ in a $1-\mathrm{Hz}$ bandwidth in units of $\mathrm{dBc}(1 \mathrm{~Hz})$, i.e., the number of decibels below the carrier in a $1-\mathrm{Hz}$ bandwidth. The noise sidebands at the $n$th laser harmonic are given by $L_{n}(f)$, shown in Fig. 1 in Ref. 6, which is the number of decibels below the carrier ( $n$th laser harmonic) of the noise sideband at a frequency offset $f$ for a given resolution bandwidth $B$. $\mathrm{L}_{1}(f)$ is then determined by $L_{n}(f)$ divided by the resolution bandwidth $B$ (more accurately the equivalent noise bandwidth) and the square of the laser harmonic $n^{2}$. The rms timing jitter $\sigma_{J}$ in a certain frequency bandwidth is then

$$
\sigma_{J}=\frac{1}{2 \pi f_{L}}\left[2 \int_{f_{\text {low }}}^{f_{\text {high }}} L_{1}(f) \mathrm{d} f\right]^{1 / 2},
$$


where $f_{L}$ is the laser first harmonic (the laser repetition rate). The rms value of the timing jitter is not based on any specific statistical distribution. If we assume, e.g., Gaussian statistics, the FWHM of the pulse arrival time distribution is given by $\approx 2.355$ times $\sigma_{J}$.

The PM NaCl color-center laser, pumped by a cw Nd:YAG laser, typically generates 275 -fsec pulses with a tunability over 1.5-1.7 $\mu \mathrm{m}$ using different semiconductor saturable absorbers. The APM KCl:Tl color-center laser, synchronously pumped by a cw modelocked Nd:YLF laser and actively controlled by a nonlinear phase retardation in the coupled cavity, typically generates 150 -fsec pulses with a tunability over 1.48-1.55 $\mu \mathrm{m}$. An APM NaCl color-center laser also produced pulse durations in the 150 -fsec regime. ${ }^{10}$ The coupled cavity of the AMP color-center laser contains a $30-\mathrm{cm}$-long single-mode, dispersion-shifted $\left(\lambda_{0}\right.$ $=1.55 \mu \mathrm{m}$ ) fiber, and its cavity length is actively controlled by a piezoelectric crystal at the end mirror. In both laser systems, a $30-\mathrm{dB}$ optical isolator is used after the pump laser.

Curve (a) in Fig. 1 shows the amplitude noise of the PM color-center laser at the shorter-wavelength range of the tuning curve. The noise is entirely dominated by the cw Nd:YAG pump laser [curve (b)] and rolls off to shot noise after approximately $200 \mathrm{kHz}$. The strong spike near $60 \mathrm{kHz}$ is the relaxation oscillation of the Nd:YAG laser. Strong, high-frequency (of a few megahertz, not shown in Fig. 1) noise spikes were observed owing to mixing of the mode beating frequency of the $\mathrm{cw} N \mathrm{Nd}$ :YAG pump laser and the repetition rate of the PM laser. This was first observed and explained for another cw-pumped, PM laser: a colliding-pulse mode-locked (CPM) dye laser pumped by a cw Ar-ion laser. ${ }^{11}$ Introduction of an intracavity étalon in the Ar-ion laser, to force single-axial-mode operation, removed these noise spikes in the CPM laser. ${ }^{12}$ It is expected that the same applies to the PM colorcenter laser. By tuning the PM laser to the longerwavelength end of the tuning range with an intracavity birefringent plate using the same absorber, an abrupt

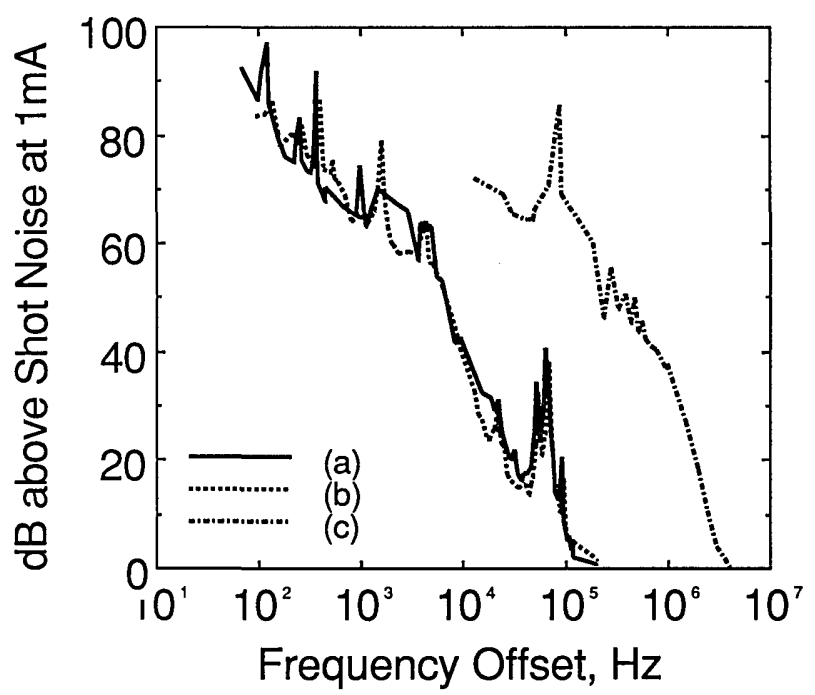

Fig. 1. Amplitude noise of the $\mathrm{PM} \mathrm{NaCl}$ color-center laser at two different operation conditions [curves (a) and (c)] and of its cw Nd:YAG pump laser [curve (b)].

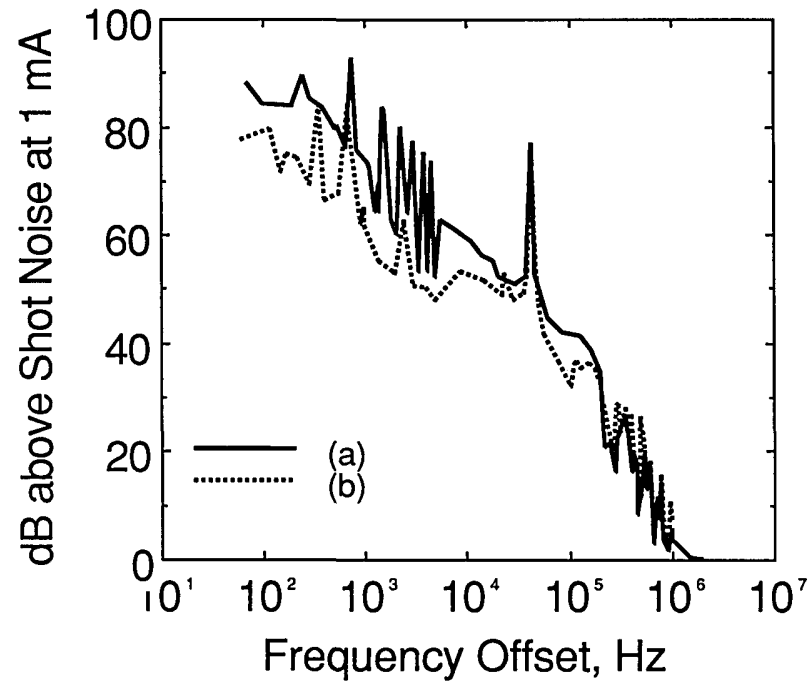

Fig. 2. Amplitude noise of the APM KCl:Tl color-center laser [curve (a)] and its cw mode-locked Nd:YLF pump laser [curve (b)].

increase of the high-frequency amplitude noise by more than $40 \mathrm{~dB}$ results [Fig. 1, curve (c)]. No abrupt changes in either the optical autocorrelation or the optical spectrum were observed. More detailed spectroscopic studies are required to understand this behavior. A 40-dB increase in amplitude noise is significant and requires a 10,000 times longer acquisition time for the same signal-to-noise ratio.

Figure 2 shows the amplitude noise of the APM color-center laser [curve (a)] in comparison with its $\mathrm{cw}$ mode-locked Nd:YLF pump laser [curve (b)]. The amplitude noise of the synchronously pumped colorcenter laser (without the nonlinear control cavity) was the same as that of the pump laser. The APM laser shows an $\approx 10$-dB increase in the amplitude noise below $200 \mathrm{kHz}$. The repetition rate of the APM colorcenter laser was $780 \mathrm{~Hz}$ offset to its pump laser's repetition rate. This introduced an amplitude modulation of the APM color-center laser pulses, which is shown in curve (a) of Fig. 2 with its harmonics. Keeping the pump laser's repetition rate fixed, we measured slightly different amplitude noise at different repetition rates of the APM laser up to an offset of $\approx 1.7 \mathrm{kHz}$. However, for all these cases at least $10 \mathrm{~dB}$ of excess noise was observed, including the case where the repetition rates of the APM laser and its pump laser were the same. Sometimes the noise spectrum became asymmetric with a distinct second peak at the repetition rate of the pump laser, which possibly implies double pulsing. The noise spike near $40 \mathrm{kHz}$ in Fig. 2 is due to the relaxation oscillation of the $\mathrm{cw}$ modelocked Nd:YLF pump laser. At higher pump powers, an unexpected, strong 10-20\% amplitude modulation near $7 \mathrm{MHz}$ appeared. In the subhertz regime drifts in power and cavity length cause the APM laser output to break up into picosecond pulses. This required periodic adjustments during measurements. In contrast, the PM laser did not lose mode locking, which typically occurred for the whole day, without any active feedback control.

The single-sided timing jitter spectral density $L_{1}(f)$ 


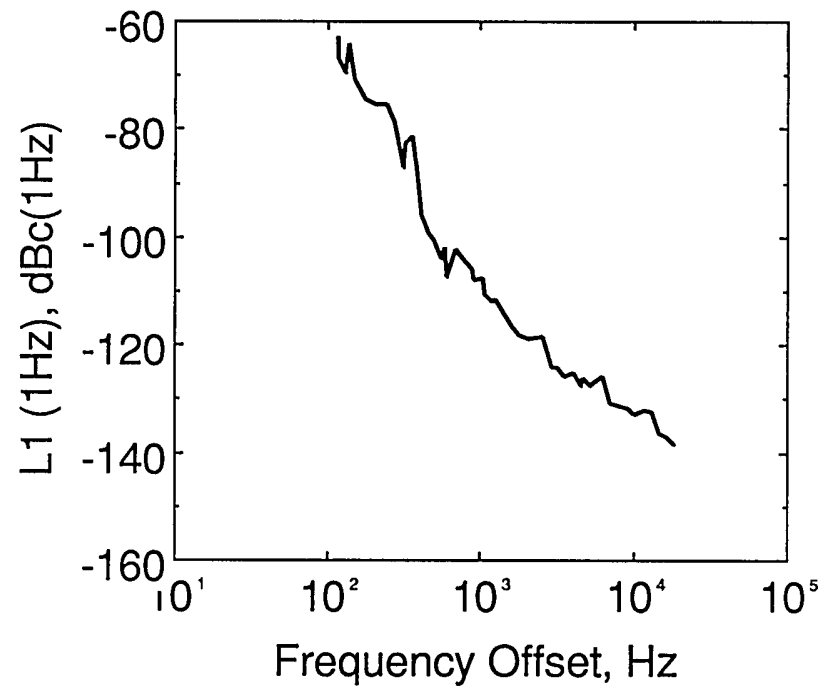

Fig. 3. Timing jitter of the $\mathrm{PM} \mathrm{NaCl}$ color-center laser, 9 psec rms above $130 \mathrm{~Hz}$.

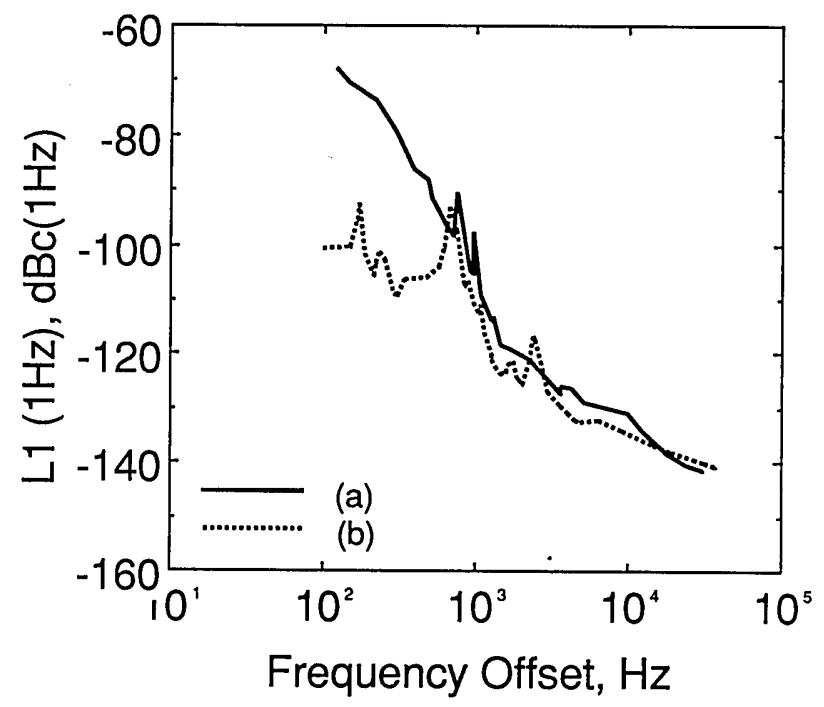

Fig. 4. Timing jitter of the APM KCl:Tl color-center laser, 9 psec rms above $130 \mathrm{~Hz}$ [curve (a)], and of its pump laser, 0.9 psec rms above $130 \mathrm{~Hz}$ [curve (b)].

in the $1-\mathrm{Hz}$ bandwidth for the PM color-center laser is shown in Fig. 3 and for the APM color-center laser is shown in Fig. 4. The rms timing jitter is directly determined by $L_{1}(f)$ [Eq. (1)] and for both lasers was approximately 9 psec in the frequency span from 130 $\mathrm{Hz}$ to $20 \mathrm{kHz}$. In comparison, curve (b) of Fig. 4 shows the $L_{1}(f)$ of the synchronously pumped KCl:Tl colorcenter laser, which was identical to the $L_{1}(f)$ of the cw mode-locked Nd:YLF pump laser. The synchronously pumped color-center laser and the $\mathrm{cw}$ mode-locked Nd:YLF pump laser had a 0.9-psec rms timing jitter, whereas the timing jitter of the APM laser was an order of magnitude higher. The significant increase in timing jitter of the APM laser is from frequencies below $1 \mathrm{kHz}$ (Fig. 4), which approximately corresponds to the bandwidth of the active feedback loop required for a useful APM laser. The active feedback loop was locked to the output power of the laser and adjusted the coupled cavity length. This is probably one reason for the large increase in timing jitter of the APM laser in comparison with its pump laser. In comparison with the $\mathrm{PM}$ color-center laser, the $L_{1}(f)$ of a CPM laser was in the same range as in Fig. $3 .{ }^{11}$ The CPM laser's overall timing jitter of 6 psec rms in the $130-\mathrm{Hz}$ to $20-\mathrm{kHz}$ frequency span was smaller owing to its higher $100-\mathrm{MHz}$ repetition rate. However, in contrast to the PM color-center laser, which is an all-solid-state laser, the CPM laser was isolated inside a box and on a floating laser table. The significant timing jitter for these lasers arises from frequencies below $1 \mathrm{kHz}$. Therefore it should be possible to improve the timing jitter of the free-running PM lasers by actively controlling the cavity length with a feedback loop system.

In conclusion, the overall noise performance of the PM laser is substantially better than that of the APM color-center laser. Over most of the wavelength tuning range the amplitude noise was entirely dominated by its pump laser, as previously observed with the CPM laser. ${ }^{12}$ The APM laser showed $>10 \mathrm{~dB}$ increased amplitude noise over a wide frequency range and, additionally, was breaking up in the subhertz regime, requiring periodic readjustment of the laser during our measurements. Both laser systems had a 9-psec rms timing jitter above $130 \mathrm{~Hz}$. However, the timing jitter of the APM laser was probably enhanced by the active feedback control system required for usable pulses.

Note added in proof: Recently, it came to our attention that Finch et al..$^{13}$ published their noise characterization of an APM KCl:Tl color-center laser.

\section{References}

1. M. N. Islam, E. R. Sunderman, C. E. Soccolich, I. BarJoseph, N. Sauer, T. Y. Chang, and B. I. Miller, IEEE J. Quantum Electron. 25, 2454 (1989).

2. L. F. Mollenauer and R. H. Stolen, Opt. Lett. 9, 13 (1984).

3. P. N. Kean, X. Zhu, D. W. Crust, R. S. Grant, N. Langford, and W. Sibbett, Opt. Lett. 14, 39 (1989).

4. J. Mark, L. Y. Liu, K. L. Hall, H. A. Haus, and E. P. Ippen, Opt. Lett. 14, 48 (1989).

5. D. von der Linde, Appl. Phys. B 39, 201 (1986).

6. U. Keller, K. D. Li, M. Rodwell, and D. M. Bloom, IEEE J. Quantum Electron. 25, 280 (1989).

7. M. J. W. Rodwell, D. M. Bloom, and K. J. Weingarten, IEEE J. Quantum Electron. 25, 817 (1989).

8. K. J. Weingarten, M. J. W. Rodwell, and D. M. Bloom, IEEE J. Quantum Electron. 24, 198 (1988).

9. Ref. 8, Eqs. (20)-(24).

10. C. P. Yakymyshyn, J. F. Pinto, and C. R. Pollock, in Digest of Conference on Lasers and Electro-Optics (Optical Society of America, Washington, D.C., 1989), paper FQ3.

11. G. T. Harvey, M. S. Heutmaker, P. R. Smith, M. C. Nuss, U. Keller, and J. A. Valdmanis, "Timing jitter and pump-induced spurious modulation in the collidingpulse mode-locked laser," IEEE J. Quantum Electron. (to be published).

12. M. C. Nuss, U. Keller, G. T. Harvey, M. S. Heutmaker, and P. R. Smith, "50-dB amplitude noise reduction of colliding-pulse mode-locking dye lasers," Opt. Lett. (to be published).

13. A. Finch, X. N. Zhu, P. N. Kean, and W. Sibbett, IEEE J. Quantum Electron. 26, 1114 (1990). 